\title{
New Methods for Modeling Laterolog Resistivity Corrections
}

\author{
Jadwiga Anna JARZYNA ${ }^{1}$, Adam CICHY ${ }^{1}$, Dezső DRAHOS ${ }^{2}$, \\ Attila GALSA ${ }^{2}$, Maria Joanna BAŁA ${ }^{1}$, and Andrzej OSSOWSKI ${ }^{1}$ \\ ${ }^{1}$ AGH University of Science and Technology, Faculty of Geology, \\ Geophysics and Environment Protection, Kraków, Poland; \\ e-mails: jarzyna@agh.edu.pl (corresponding author), cichy@agh.edu.pl, \\ bala@geol.agh.edu.pl,ossowski@geol.agh.edu.pl \\ ${ }^{2}$ Eötvös Loránd University, Department of Geophysics and Space Sciences, \\ Budapest, Hungary; e-mails: drahos@pangea.elte.hu, gali@pangea.elte.hu
}

\section{Abstract}

The paper presents methods for laterolog response modeling. In Coulomb's charges method, Laplace's equation is solved for the electric field distribution in rock medium with internal boundaries between different resistivity layers. There, the boundary problem is reduced to Fredholm integral equation of the second kind. The second method uses a finite element array to model apparent resistivity from laterolog. The task is treated as DC problem and the Laplace equation is solved numerically. The presented methods were applied to borehole data covering a typical stratigraphic section of the Fore-Sudetic Monocline in southwestern Poland. Apparent resistivity was calculated using the Coulomb's charges method and alternatively modeled using a finite element method which gave similar results. Then, a series of linear corrections for borehole, shoulder bed, and filtration effects for apparent resistivity obtained by the Coulomb's charges method demonstrated the feasibility of calculating true resistivity of virgin and invaded zones. The proposed methods provide a flexible solution in modeling which can be adapted to other logs.

Key words: laterolog response, Coulomb's charges method, finite element modeling, apparent resistivity correction. 


\section{INTRODUCTION}

The modeling of resistivity response observed from various resistivity logs employs analytical and numerical methods that come with tradeoffs. Analytical methods may require numerical solutions such as series representation of Bessel functions or numerical evaluation of integrals. Geologic structures and invasion zone features around the borehole may complicate analytical solutions. Even with recent advances in computational power, analytical methods can only be applied to simple 1D or 2D rock formation models (Drahos 1984, Anderson 2001).

Numerical solutions lend themselves more readily to modeling resistivity in complicated geologic media. They have been adapted to 2D and 3D examples of logging environments, but depend on finite difference or finite element routines that generate a large number of computationally intensive linear equations. Fast computers, if available, can easily solve these equations.

Laterolog electrode tools can be modeled using relatively simple analytical expressions and numerical solutions. Attempts have been made to numerically correct for the Groningen effect on LLD resistivity (Lovell 1993). Potential formulation based on a Poisson/Laplace equation was shown to be effective. The shape and size of electrodes present challenges in calculating laterolog resistivity responses and numerical modeling, however, and thus require certain assumptions that may affect results.

A large body of computer code has been developed to model resistivity response (Anderson 2001). Methods are based on analytical (Moran and Timmons 1957) as well as numerical solutions (Nam et al. 2010). Several of these methods are described here as relevant examples of efforts to improve resistivity models. Wang et al. (1998) designed LL3D, a 3D finite element code for computing laterolog response in arbitrary geometries. A later version of this code addressed anisotropic media. Davydycheva et al. (1996) developed the DC3D method, a 3D finite difference tool for computing laterolog response in a complicated geometry. This approach uses impedance boundary conditions and improves accuracy in cases of high resistivity contrasts through material averaging (Moskow et al. 1999).

The variety of algorithms for resistivity modeling arises not only from differences in numerical methods but also from specific approach to geological model performance from performance requirements of different geologic environments (Ribeiro and Carrasquilla 2013). The above-mentioned authors included a polynomial mathematical representation of an invaded zone having variable diameter and resistivity. A simplified method of resistivity modeling in complicated geologic structures (multiple beds, boreholes, and invaded zones) used a general recursive algorithm and local reflection and 
transmission operators from a single bed boundary (Chew et al. 1991). In this method, computational requirements scaled linearly, rendering the method more efficient than the finite element approach to the same problem. Another approach was based on optimization of the finite element $h p$ mesh where $h$ was the size of the finite element and $p$ was the polynomial element order of approximation (Pardo et al. 2005, Nam et al. 2010).

The above summary of analytical solutions and numerical modeling demonstrates the state of the art as well as the significance of further efforts to improve resistivity data interpretation from dual laterolog measurements. The results of two different approaches to dual laterolog resistivity modeling are presented below. These methods build on previous work and can be adapted to future refinement of resistivity analysis.

\section{DUAL LATEROLOG RESISTIVITY MEASUREMENT}

Dual laterolog techniques use nine electrodes operating according to galvanic conduction principles. LLD and LLS refer to respective deep and shallow operating modes of the device. These measurements are a popular and still viable version of multielectrode laterologs (Ellis and Singer 2007). Lowfrequency alternating currents $(\mathrm{AC})$ are used to reduce contact-impedance electrical noise and interference from electromagnetic fields. The log is typically considered a direct current (DC) measurement, however (Ellis and Singer 2007, Nam et al. 2010). Total current emitted by the laterolog LLD device returns to the torpedo (the lower section of the armored cable) and the $\mathrm{N}$ electrode (located on the bridle, an insulated cable run between torpedo and the top of the device) provides a reference voltage reading. When the torpedo and device enter a resistive unit, the $\mathrm{N}$ electrode voltage becomes negative (Trouiller and Dubourg 1994). For proper resistivity recording, the reference voltage should equal zero. It can become negative due to skin effects from the $35 \mathrm{~Hz} \mathrm{AC}$ of the device, due to the presence of a high resistivity unit above a lower resistivity unit, or due to steel casing apparatus. Reference electrode effects have always appeared in LLD measurements and are referred to as the Delaware and Groningen effects. They cause an increase in apparent resistivity of otherwise low resistivity beds. A number of technical fixes, including additional electrodes are used to reduce these effects, but their resultant noise and uncertainties persist.

This paper considers the DLT-GA dual laterolog device made by Halliburton Co. (Halliburton 1992) and compares both LLD and LLS operating modes.

Well log analysts have encountered the problem of elevated apparent resistivity in trying to interpret a Permian unit referred to as the Main Dolomite, which appears in cores and boreholes from the Fore-Sudetic Monocline of southwestern Poland. This unit exhibits medium and low apparent resis- 
tivity occurring beneath a high resistivity unit referred to as the Basal Anhydrite (Table 2). Apparent resistivity of a typical section observed in borehole data from the Fore-Sudetic Monocline was calculated using the Coulomb's charges method (Cichy and Ossowski 2015). Drahos and Galsa (2007) solved the same problem using finite element method. Results from the two different approaches are compared below. Similarities in apparent resistivity datasets from each method were interpreted as a successful correctness test.

\section{COULOMB'S CHARGES METHOD FOR MODELING RESISTIVITY}

In the Coulomb's charges method, electric field distribution within rock media having internal boundaries between horizons of different resistivity is obtained by solving the Laplace equation with the following internal boundary conditions:

$$
\begin{aligned}
\frac{1}{r} \frac{\partial}{\partial r}\left(\frac{r}{R} \frac{\partial V}{\partial r}\right)+\frac{\partial}{\partial z}\left(\frac{1}{R} \frac{\partial V}{\partial z}\right)=0, & \frac{1}{R^{i}} E_{n}^{i}(r, z)=\frac{1}{R^{i+1}} E_{n}^{i+1}(r, z), \\
E & =-\nabla V .
\end{aligned}
$$

where $V$ is the electric field potential, $R^{i}$ and $R^{i+1}$ are resistivities of homogenous layers $i$ and $i+1 ; \quad E_{n}^{i}(r, z)$ and $E_{n}^{i+1}(r, z)$ are normal components of the electrical field at boundaries separating the $i$ and $i+1$ domains, and $r$ and $z$ are coordinates of the cylindrical system.

Surface electric charge density $\sigma(r, z)$ was calculated according to the formula:

$$
\sigma(r, z)=2 \varepsilon_{0} K(r, z) E_{n}^{\mathrm{av}}(r, z),
$$

where $K(r, z)=\left(R^{i+1}-R^{i}\right) /\left(R^{i+1}+R^{i}\right) \quad$ is the reflectivity coefficient, $E_{n}^{\mathrm{av}}(r, z)=\left(E_{n}^{i}(r, z)+E_{n}^{i+1}(r, z)\right) / 2$ is an average of the electric field normal components, and $\varepsilon_{0}$ is the permittivity of free space.

The normal component of the electric field, $E_{n}^{\text {av }}(r, z)$, is a sum of components related to primary sources, $E_{n}^{s c}(r, z)$, charge induced on horizontal boundaries between layers, $E_{n}^{h}(r, z)$, charge induced on cylindrical boundaries, $E_{n}^{c}(r, z)$, and charge from cylindrical electrodes of the device, $E_{n}^{s}(r, z)$,

$$
E_{n}^{\mathrm{av}}(r, z)=E_{n}^{s c}(r, z)+E_{n}^{h}(r, z)+E_{n}^{c}(r, z)+E_{n}^{s}(r, z) .
$$

The boundary problem for the Laplace equation with internal boundary conditions reduces to the Fredholm integral equation of the second kind (Alpin 1964, Alpin et al. 1985, Cichy and Ossowski 2015). 


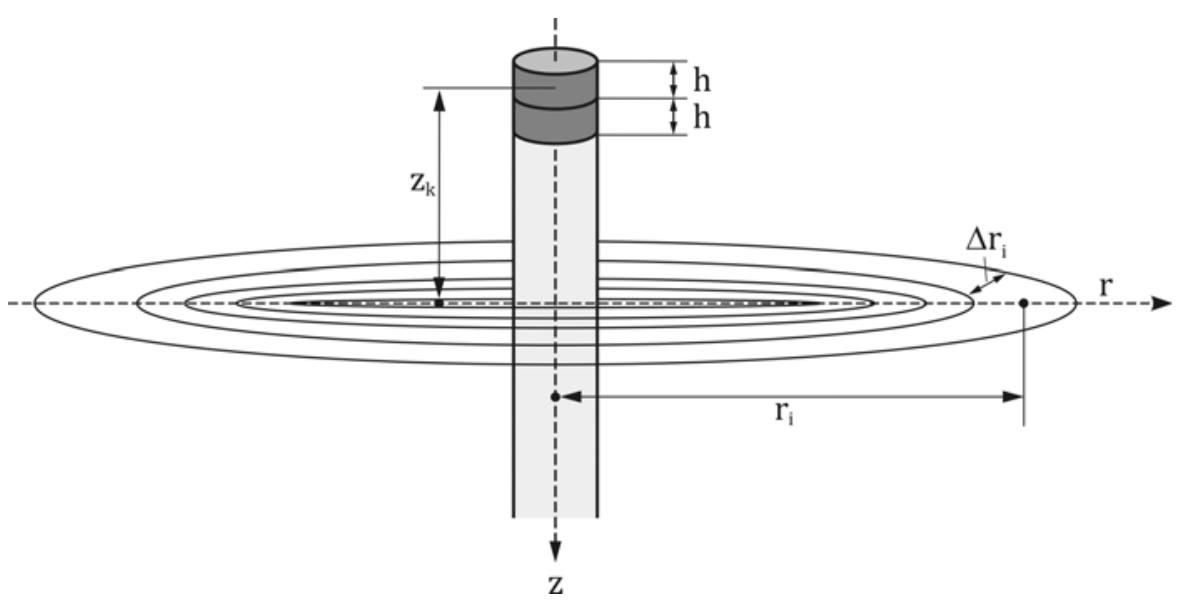

Fig. 1. Diagram showing cylindrical and horizontal boundaries for elementary cylinders and rings (after Cichy and Ossowski 2014).

In the next step, horizontal and cylindrical boundaries between layers of the rock medium that vary in terms of resistivity were discretized as elementary cylinders, rings, and horizontal layers (Fig. 1). The method then determines a set of linear equations with unknowns defined as charge density on the elementary units. The electric potential at any point in the borehole and rock formation from all current sources and charges on all elements (cylinders and horizontal surfaces) is calculated as a superposition.

The size of the model domain was determined empirically. The maximum space for calculation was determined for $z$ values of up to $50 \mathrm{~m}$ and $r$ values of up to $230 \mathrm{~m}$. The constructed vertical cylinder approximated a borehole of $0.20 \mathrm{~m}$ diameter, with height extending both $25 \mathrm{~m}$ above the laterolog's central electrode A0, and $25 \mathrm{~m}$ below. The borehole was divided into elementary cylinders of $0.20 \mathrm{~m}$ diameter and $0.05 \mathrm{~m}$ height. Calculations addressed one thousand of such cylinders within the $50 \mathrm{~m}$ vertical section of the borehole. Each horizontal boundary consisted of 500 rings. The size, $\Delta r_{i}$, of the first ring and the next 249 ones was fixed at $0.05 \mathrm{~m}$. The sizes, $\Delta r_{i}$, of the proceeding 250 rings increased in geometric progression by a factor of about 1.02. Iterative testing identified optimal size, height, and diameter parameters for the model. This discretization of the model allowed us to solve the system of linear equations to within $2 \%$ accuracy of calculations (Cichy and Ossowski 2015). The accuracy was determined by comparing results of the Coulomb's charges calculation with those of the analytical solution described in Alpin et al. (1985). 


\section{LATEROLOG RESPONSE}

Electric current and potential were calculated as a superposition of appropriate signals from normal logs. Single normal log response was calculated in the presence of all cylindrical electrodes of the laterolog device in the borehole when only one electrode was supplied with current, $I_{0}$ (Fig. 2). Resistivity of the cylindrical electrodes was $10^{-5} \mathrm{ohmm}$. The size of cylindrical electrodes was set according to specification of the device's Technical Manual (Halliburton 1992). The device's trunk material was assumed to be an isolator. Electrical charges generated on the trunk surface were not included in the sum of electrical field components (Eq. 3). The method thus determined the influence of all electrodes and each differentiated element of the rock formation on electric field generation.

Currents listed in Fig. 2 caption satisfy Eqs. 4 and enabled proper functioning of the laterolog.

$$
I_{1}=I_{\mathrm{A} 1}+I_{\mathrm{Al}^{\prime}}, \quad \text { and } \quad I_{2}=I_{\mathrm{A} 2}+I_{\mathrm{A} 2^{\prime}},
$$

Table 1 gives current, potential and geometric conditions for the DLTGA laterolog device.

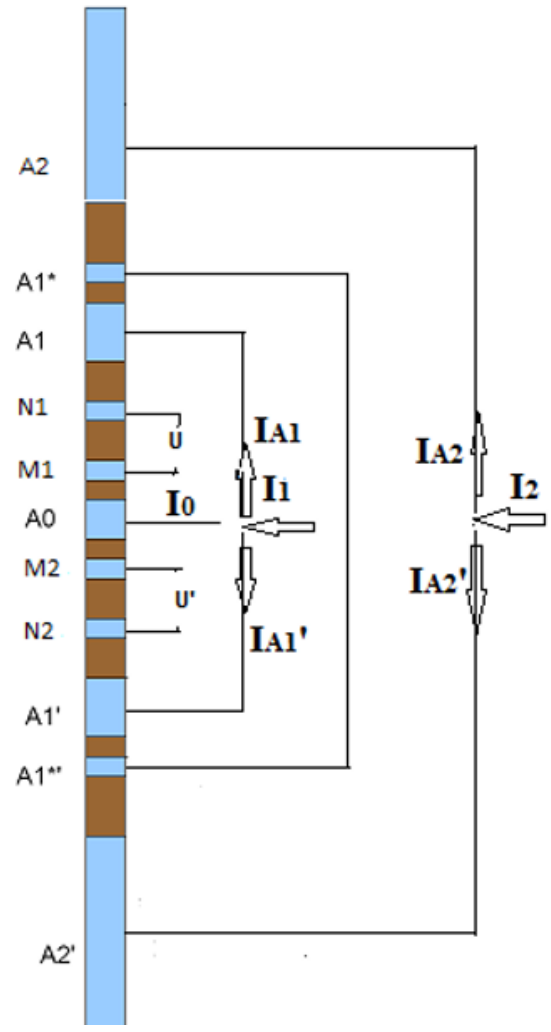

Fig. 2. Model scheme for the DLT-GA laterolog device (Halliburton 1992): A0, $\mathrm{A} 1, \mathrm{~A} 1^{\prime}, \mathrm{A} 2, \mathrm{~A} 2^{\prime}$ - current electrodes; $\mathrm{A} 1^{*}, \mathrm{~A} 1^{*}$ - monitoring electrodes; $\mathrm{M} 1$, M2, N1, N2 - measuring electrodes; $U$, $U^{\prime}$ - voltage between pair of electrodes $\mathrm{M} 1$ and N1, and between M2 and N2, respectively; $I_{0}, I_{1}, I_{\mathrm{A} 1}, I_{\mathrm{A} 1}{ }^{\prime}, I_{2}, I_{\mathrm{A} 2}, I_{\mathrm{A} 2}{ }^{\prime}-$ currents flowing through respective electrodes $\mathrm{A} 0, \mathrm{~A} 1, \mathrm{~A} 1^{\prime}, \mathrm{A} 2, \mathrm{~A} 2^{\prime} ; \mathrm{A} 1, \mathrm{~A} 1^{\prime}-$ short connected; A2, A2' - short connected; $\mathrm{Al}^{*}, \mathrm{~A} 1^{*}$ - - short connected; M1, M2 - not connected; N1, N2 - not connected. 
Conditions for the DLT-GA device according to Fig. 2

\begin{tabular}{|c|c|}
\hline LLD & LLS \\
\hline $\begin{array}{l}\text { Currents } I_{1} \text { and } I_{2} \text { adjusted so that the } \\
\text { measured electrode potentials satisfy the } \\
\text { equation: } \\
\qquad\left(V_{\mathrm{N} 1}+V_{\mathrm{N} 2}\right)-\left(V_{\mathrm{M} 1}+V_{\mathrm{M} 2}\right)=0 \\
\text { Current } I_{2} \text { adjusted so that all potentials } \\
\text { are equal: } \\
\qquad V_{\mathrm{A} 1}=V_{\mathrm{A} 2}=V_{\mathrm{Al}^{\prime}}=V_{\mathrm{A} 2^{\prime}} \\
\text { Current lines discharged from the A } \\
\text { electrodes are closed to the return } \mathrm{B} \\
\text { electrode located at the surface or within } \\
\text { the borehole (fish) so as to satisfy the } \\
\text { equation: } \\
\qquad I_{\mathrm{B}}=I_{\mathrm{A} 0}+I_{\mathrm{A} 1}+I_{\mathrm{A} 1^{\prime}}+I_{\mathrm{A} 2}+I_{\mathrm{A} 2^{\prime}} \\
\text { Potential is measured by electrodes } \mathrm{M} 1 \text {, } \\
\text { N1, } \mathrm{M} 1^{\prime}, \mathrm{N} 1^{\prime}: \\
V^{*}=1 / 4\left(V_{\mathrm{M} 1}+V_{\mathrm{M} 2}+V_{\mathrm{N} 1}+V_{\mathrm{N} 2}\right) \\
\text { Reference electrode } \mathrm{N} \text { resides } 80 \mathrm{ft} \\
\text { beneath the torpedo. }\end{array}$ & $\begin{array}{l}\text { Current } I_{1} \text { adjusted so that the meas- } \\
\text { ured electrode potentials satisfy the } \\
\text { equation: } \\
\qquad\left(V_{\mathrm{N} 1}+V_{\mathrm{N} 2}\right)-\left(V_{\mathrm{M} 1}+V_{\mathrm{M} 2}\right)=0 \\
\text { Current } I_{2}=I_{\mathrm{A} 0}+I_{\mathrm{A} 1}+I_{\mathrm{Al}^{\prime}} \\
\text { Potential is measured by electrodes } \\
\mathrm{M} 1, \mathrm{~N} 1, \mathrm{M} 2, \mathrm{~N} 2 \text { : } \\
\qquad V^{*}=1 / 4\left(V_{\mathrm{M} 1}+V_{\mathrm{M} 2}+V_{\mathrm{N} 1}+V_{\mathrm{N} 2}\right) \\
\text { Reference electrode } \mathrm{N} \text { resides } 80 \mathrm{ft} \\
\text { beneath the torpedo. }\end{array}$ \\
\hline
\end{tabular}

Potentials at measuring (detector) electrodes M1, M2, N1, N2 are a linear combination of potentials measured by normal devices of suitable length:

$$
\begin{gathered}
V_{\mathrm{M} 1}=\beta V_{\mathrm{A} 1 \mathrm{M} 1}^{0}+\gamma V_{\mathrm{A} 1^{\prime} \mathrm{M} 1}^{0}+\delta V_{\mathrm{A} 2 \mathrm{M} 1}^{0}+\lambda V_{\mathrm{A} 2^{\prime} \mathrm{M} 1}^{0}+V_{\mathrm{A} 0 \mathrm{M} 1}^{0}, \\
V_{\mathrm{N} 1}=\beta V_{\mathrm{A} 1 \mathrm{~N} 1}^{0}+\gamma V_{\mathrm{A} 1^{\prime} \mathrm{N} 1}^{0}+\delta V_{\mathrm{A} 2 \mathrm{~N} 1}^{0}+\lambda V_{\mathrm{A} 2^{\prime} \mathrm{N} 1}^{0}+V_{\mathrm{A} 0 \mathrm{~N} 1}^{0}, \\
V_{\mathrm{M} 2}=\beta V_{\mathrm{A} 1 \mathrm{M} 2}^{0}+\gamma V_{\mathrm{A} 1^{\prime} \mathrm{M} 2}^{0}+\delta V_{\mathrm{A} 2 \mathrm{M} 2}^{0}+\lambda V_{\mathrm{A} 2^{\prime} \mathrm{M} 2}^{0}+V_{\mathrm{A} 0 \mathrm{M} 2}^{0}, \\
V_{\mathrm{N} 2}=\beta V_{\mathrm{A} 1 \mathrm{~N} 2}^{0}+\gamma V_{\mathrm{A} 1^{\prime} \mathrm{N} 2}^{0}+\delta V_{\mathrm{A} 2 \mathrm{~N} 2}^{0}+\lambda V_{\mathrm{A} 2^{\prime} \mathrm{N} 2}^{0}+V_{\mathrm{A} 0 \mathrm{~N} 2}^{0},
\end{gathered}
$$

where $V_{\mathrm{A}_{i} \mathrm{M} 1}^{0}, V_{\mathrm{A}_{i} \mathrm{M} 2}^{0}, V_{\mathrm{A}_{i} \mathrm{~N} 1}^{0}, V_{\mathrm{A}_{i} \mathrm{~N} 2}^{0}$ are potentials measured by normal logs of length $\mathrm{A}_{i} \mathrm{M} 1, \mathrm{~A}_{i} \mathrm{M} 2, \mathrm{~A}_{i} \mathrm{~N} 1, \mathrm{~A}_{i} \mathrm{~N} 2$, where $i$ refers to each electrode in sequence $(i=0,1,2)$. Normal $\operatorname{logs}$ were supplied with $I_{0}$ current.

Multiplication factors, $\beta, \gamma, \delta$, and $\lambda$ (Eq. 6), represent ratios of currents $I_{\mathrm{A} 1}, I_{\mathrm{A} 1^{\prime}}, I_{\mathrm{A} 2}$, and $I_{\mathrm{A} 2^{\prime}}$ as discharged from electrodes $\mathrm{A}_{1}, \mathrm{~A}_{1^{\prime}}, \mathrm{A}_{2}$, and $\mathrm{A}_{2^{\prime}}$ to current $I_{0}$ flowing from the central electrode $\mathrm{A}_{0}$. 


$$
\beta=\frac{I_{\mathrm{A} 1}}{I_{0}}, \quad \gamma=\frac{I_{\mathrm{Al}^{\prime}}}{I_{0}}, \quad \delta=\frac{I_{\mathrm{A} 2}}{I_{0}}, \quad \lambda=\frac{I_{\mathrm{A} 2^{\prime}}}{I_{0}},
$$

In the case of LLD mode laterolog in non-homogenous rock media, multiplication factors for currents $I_{1}$ and $I_{2}$ (Eq. 4) (Table 2) served to equalize potentials on measuring electrodes and were calculated using Eqs. 7-10:

$$
\begin{aligned}
& \beta=\alpha g_{\mathrm{A} 1}, \quad \gamma=\alpha g_{\mathrm{Al}^{\prime}}, \quad \delta=\alpha w g_{\mathrm{A} 2}, \quad \lambda=\alpha w g_{\mathrm{A}^{\prime}},
\end{aligned}
$$

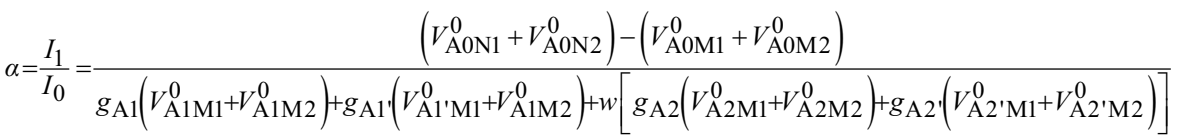

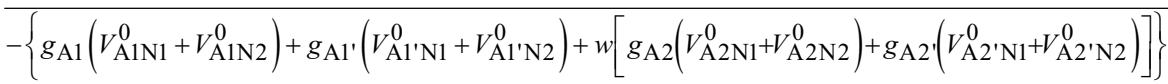

$$
\begin{aligned}
& w=\frac{I_{2}}{I_{1}}=\frac{g_{\mathrm{A} 1} V_{\mathrm{A} 1}^{0}}{g_{\mathrm{A} 2} V_{\mathrm{A} 2}^{0}}, \\
& g_{\mathrm{A} 1}=\frac{I_{\mathrm{A} 1}}{I_{1}}=\frac{V_{\mathrm{A} 1^{\prime}}^{0}}{V_{\mathrm{A} 1}^{0}+V_{\mathrm{A} 1^{\prime}}^{0}}, \quad g_{\mathrm{A} 2}=\frac{I_{\mathrm{A} 2}}{I_{2}}=\frac{V_{\mathrm{A} 2^{\prime}}^{0}}{V_{\mathrm{A} 2}^{0}+V_{\mathrm{A} 2^{\prime}}^{0}}, \\
& g_{\mathrm{Al}^{\prime}}=\frac{I_{\mathrm{Al}^{\prime}}}{I_{1}}=\frac{V_{\mathrm{A} 1}^{0}}{V_{\mathrm{A} 1}^{0}+V_{\mathrm{A}^{\prime}}^{0}}, \quad g_{\mathrm{A}^{\prime}}=\frac{I_{\mathrm{A} 2^{\prime}}}{I_{2}}=\frac{V_{\mathrm{A} 2}^{0}}{V_{\mathrm{A} 2}^{0}+V_{\mathrm{A}^{\prime}}^{0}} .
\end{aligned}
$$

Relevant multiplication factors for LLS mode currents are defined by the Eqs. 11-12.

$$
\begin{aligned}
& \beta=\alpha g_{\mathrm{A} 1}, \quad \gamma=\alpha g_{\mathrm{Al}^{\prime}}, \quad \delta=(1-\alpha) g_{\mathrm{A} 2}, \quad \lambda=(1-\alpha) g_{\mathrm{A} 2^{\prime}}, \quad w=-1,
\end{aligned}
$$

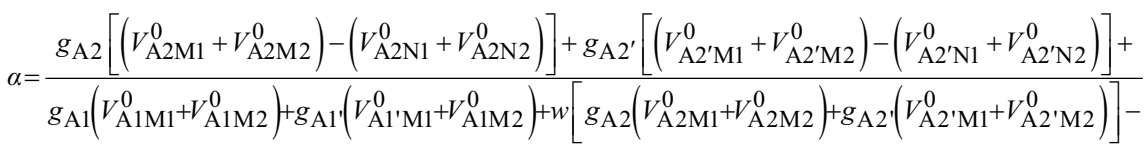

$$
\begin{aligned}
& \left(V_{\mathrm{A} 0 \mathrm{~N} 1}^{0}+V_{\mathrm{A} 0 \mathrm{~N} 2}^{0}\right)-\left(V_{\mathrm{A} 0 \mathrm{M} 1}^{0}+V_{\mathrm{A} 0 \mathrm{M} 2}^{0}\right)
\end{aligned}
$$

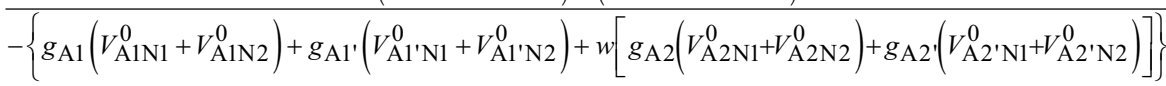

Potential measured in LLD and LLS modes was calculated according to Eq. 13 while apparent resistivity was determined according to Eq. 14.

$$
V^{*}=1 / 4\left(V_{\mathrm{M} 1}+V_{\mathrm{M} 2}+V_{\mathrm{N} 1}+V_{\mathrm{N} 2}\right),
$$



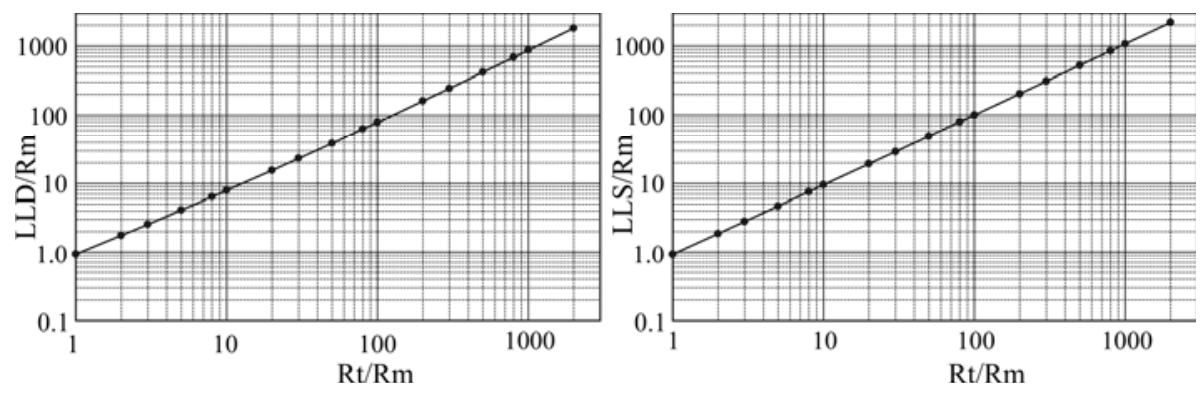

Fig. 3. Apparent resistivity versus true resistivity in a homogenous rock section sampled from a borehole of $0.20 \mathrm{~m}$ diameter with mud resistivity of $R_{m}=1 \mathrm{ohm} \mathrm{m}$. Results on the left are from LLD mode, results on the right from LLS mode.

$$
R_{a}=K \frac{V^{*}}{I_{0}} .
$$

Geometric coefficient values were adopted from the DLT-GA device Technical Manual as $K^{\mathrm{LLD}}=0.81 \mathrm{~m}$ and $K^{\mathrm{LLS}}=1.45 \mathrm{~m}$.

Test calculations were performed using a homogenous rock section sampled from a borehole of $0.2 \mathrm{~m}$ diameter with mud resistivity $R_{m}=1 \mathrm{ohm} \mathrm{m}$. The tests gave rock formation resistivities of $1,2,3,5,8,10,20,30,50,80$, $100,200,300,500,800,1000$, and $2000 \mathrm{ohm} \mathrm{m}$. Figure 3 shows the results of apparent resistivity calculations.

\section{ELTE METHOD FOR RESISTIVITY MODELING}

The finite element software package, COMSOL Multiphysics v. 4.2a, was used to model the apparent resistivity measured in LLD mode for an inhomogeneous medium. The task was framed as a DC problem due to the low frequency of device measuring conditions, allowing the Laplace Eq. 1 to be solved numerically.

Figure 4 shows finite element implementation of the deep penetration function of the DLT-GA dual laterolog device. Guard electrodes $\left(\mathrm{A}_{1}, \mathrm{~A}_{1}{ }^{*}\right.$, and $A_{2}$ in Fig. 2) above measuring electrodes were merged into an electrode array referred to as $A_{1}$, which was treated as a metal body (grey) with uniform potential. The surface of the device between $A_{1}$ and $A_{2}$ (Fig. 2) was treated as an insulating boundary condition (blue). The lower guard electrode was treated in a similar manner and referred to as $\mathrm{A}_{2}$. Reference electrode $\mathrm{N}$ and the return electrode $\mathrm{B}$ were positioned 25 and $125 \mathrm{~m}$, respectively, above the center of the device. Point and line current sources (red) served as respective sources for short $\left(A_{0}\right.$ and $\left.B\right)$ and long $\left(A_{1}\right.$ and $\left.A_{2}\right)$ current electrodes. 


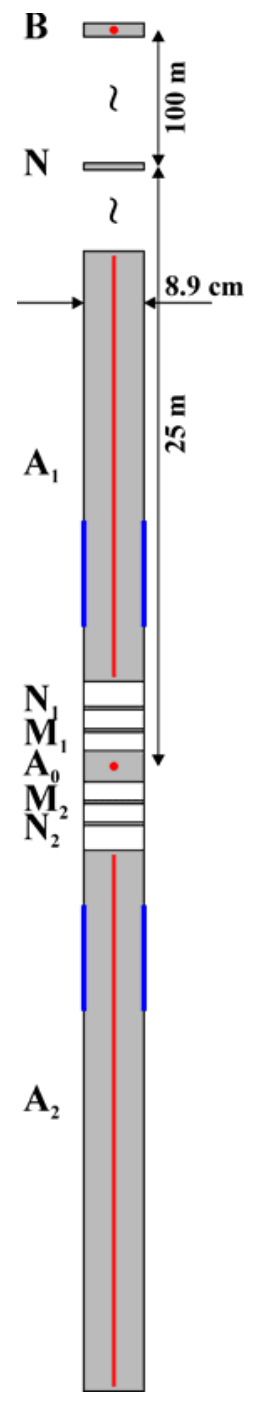

Fig. 4. Physical model $(5 \times$ horizontal enlargement $)$ on which the deep penetration function of the DLT-GA dual laterolog is based. Grey color denotes metal apparatus assumed to have a resistivity of $10^{-5} \mathrm{ohm} \mathrm{m}$, white is an insulator with resistivity of $10^{5} \mathrm{ohmm}$, blue indicates insulating boundary conditions, and red denotes current sources.

The superposition theorem was used to ensure the potential equivalence between measuring electrodes $\mathrm{M}_{1}$ and $\mathrm{N}_{1}$, as well as between $\mathrm{M}_{2}$ and $\mathrm{N}_{2}$ (Fig. 4). The electrode potentials caused by individual current electrodes $A_{0}$, $\mathrm{A}_{1}, \mathrm{~A}_{2}$, and $\mathrm{B}$ were calculated as the middle, two guard and return current electrodes, respectively. The guard electrode current was determined as follows. Due to the laterolog principle, potentials on electrodes $\mathrm{M}_{1}$ and $\mathrm{N}_{1}$, as well as on $\mathrm{M}_{2}$ and $\mathrm{N}_{2}$, are set to be equal: $V_{\mathrm{M} 1}=V_{\mathrm{N} 1}, V_{\mathrm{M} 2}=V_{\mathrm{N} 2}$, where

$$
V_{\mathrm{M} 1}=I_{0}\left[\tilde{R}_{\mathrm{A} 0 \mathrm{M} 1}+n_{1} \tilde{R}_{\mathrm{A} 1 \mathrm{M} 1}+n_{2} \tilde{R}_{\mathrm{A} 2 \mathrm{M} 1}-\left(1+n_{1}+n_{2}\right) \tilde{R}_{\mathrm{BM} 1}\right],
$$




$$
\begin{gathered}
V_{\mathrm{N} 1}=I_{0}\left[\tilde{R}_{\mathrm{A} 0 \mathrm{~N} 1}+n_{1} \tilde{R}_{\mathrm{A} 1 \mathrm{~N} 1}+n_{2} \tilde{R}_{\mathrm{A} 2 \mathrm{~N} 1}-\left(1+n_{1}+n_{2}\right) \tilde{R}_{\mathrm{BN} 1}\right], \\
V_{\mathrm{M} 2}=I_{0}\left[\tilde{R}_{\mathrm{A} 0 \mathrm{M} 2}+n_{1} \tilde{R}_{\mathrm{A} 1 \mathrm{M} 2}+n_{2} \tilde{R}_{\mathrm{A} 2 \mathrm{M} 2}-\left(1+n_{1}+n_{2}\right) \tilde{R}_{\mathrm{BM} 2}\right], \\
V_{\mathrm{N} 2}=I_{0}\left[\tilde{R}_{\mathrm{A} 0 \mathrm{~N} 2}+n_{1} \tilde{R}_{\mathrm{A} 1 \mathrm{~N} 2}+n_{2} \tilde{R}_{\mathrm{A} 2 \mathrm{~N} 2}-\left(1+n_{1}+n_{2}\right) \tilde{R}_{\mathrm{BN} 2}\right],
\end{gathered}
$$

and the potential on the reference electrode due to the current sources is

$$
V_{\mathrm{N}}=I_{0}\left[\tilde{R}_{\mathrm{A} 0 \mathrm{~N}}+n_{1} \tilde{R}_{\mathrm{A} 1 \mathrm{~N}}+n_{2} \tilde{R}_{\mathrm{A} 2 \mathrm{~N}}-\left(1+n_{1}+n_{2}\right) \tilde{R}_{\mathrm{BN}}\right] .
$$

In Eqs. $15 \mathrm{a}-\mathrm{d}, I_{0}$ denotes current discharged from the middle electrode $\left(\mathrm{A}_{0}\right), \tilde{R}_{i j}$ is the transfer resistance between the current electrode $i$ and the measuring electrode $j$ (i.e., the potential on electrode $j$ as a consequence of the current flowing out from electrode $i$ ), $n_{1}$ and $n_{2}$ are factors representing the guard electrode currents relative to the middle electrode current $\left(n_{1}=I_{1} / I_{0}, \quad n_{2}=I_{2} / I_{0}\right.$, where $I_{1}$ and $I_{2}$ are the currents flowing from guard electrodes $A_{1}$ and $A_{2}$, respectively). From Eqs. 15a-d, the two unknown multipliers can be obtained for an arbitrary heterogeneous medium. Multipliers calculated by the methods above ensure a focused current field for $\mathrm{A}_{0}$ and thus maximal penetration depth for the device.

A two-dimensional axial geometry was used to solve the problem. The finite element model was extensively validated by refinement of the mesh resolution, size of the model domain, and device performance at layer boundaries.

Numerical tests served to minimize the effects of mesh resolution on electrode potential. Global mesh size adjustments did not necessarily provide more exact results. Local adjustments around areas of the device that experience high current density can, however, significantly affect the accuracy of calculation (Drahos and Galsa 2007). Figure 5 shows local mesh refinements around the metal electrodes and the relative potential variation on electrodes as a function of the number of finite elements. The last three points in Fig. $5 \mathrm{~b}$ are local manual refinements. The final mesh contained 366250 finite elements with a maximum size of $1 \mathrm{~mm}$ along the surface of the device and $2 \mathrm{~mm}$ within the metal electrodes. The potentials on short current electrodes $\mathrm{A}_{0}$ and $\mathrm{B}$ varied by almost $3 \%$ due to variation in mesh unit size. The geologic medium was assumed to be homogeneous with a resistivity of $1 \mathrm{ohmm}$. Only the middle and the return electrodes discharged current, with $I_{0}=1 \mathrm{~A}$ and $I_{B}=-1 \mathrm{~A}$.

The geologic medium surrounding the device was modeled along the vertical symmetry axis of a cylinder of radius $R$ and height $H$. The outer boundary of the cylinder was grounded. A smaller cylinder surrounding the device was introduced into the model to provide finer mesh around the 

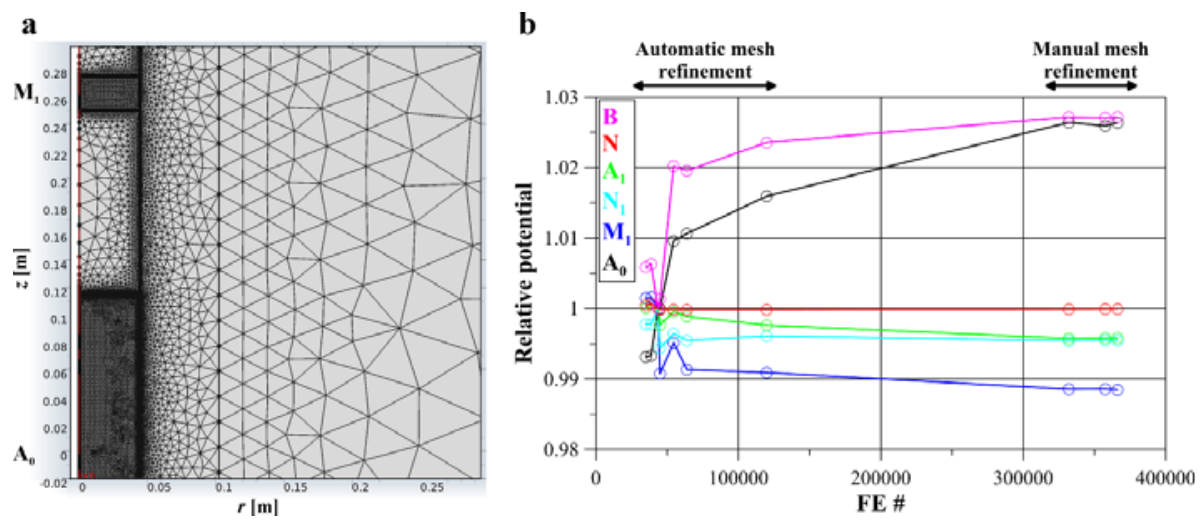

Fig. 5: (a) Finite element mesh surrounding the metal electrodes and within the borehole; (b) Effects of the number of finite elements on electrode potential in a homogeneous medium of $R_{t}=R_{m}=1 \mathrm{ohm} \mathrm{m}$. Relative potential is compared to the normal mesh in COMSOL software.

a

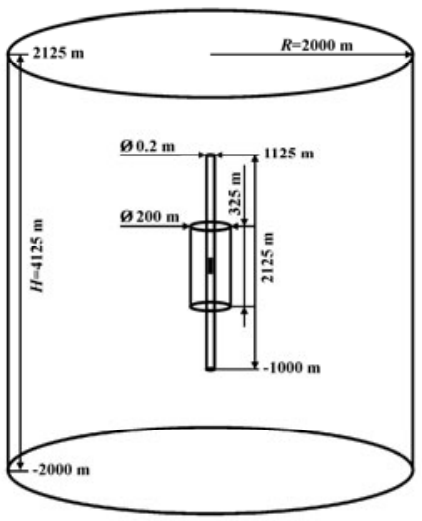

b

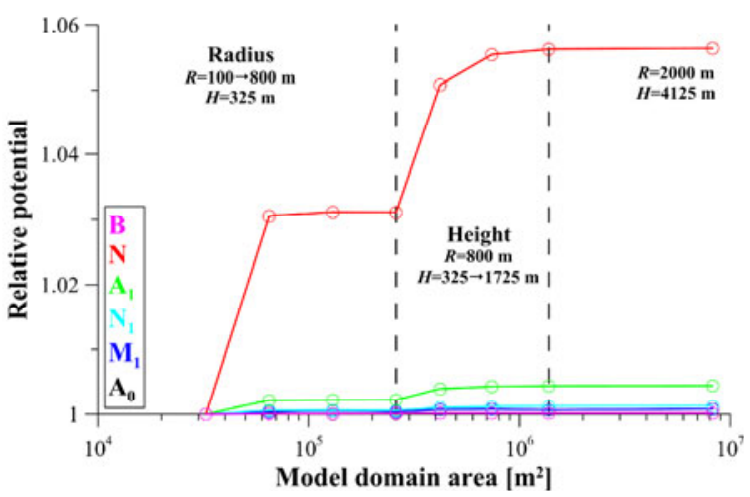

Fig. 6. Sketch of the model geometry (a) and relative potential variation of the electrodes plotted against the area of the model domain (b).

laterolog. Figure 6a shows the geometry of the model. The influence of different $R$ and $H$ values on electrode potential demonstrated effects of the model domain size. Figure $6 \mathrm{~b}$ shows the corresponding variation in electrode potentials as a function of the model domain volume in three steps: (i) the radius of the domain was increased from 100 to $800 \mathrm{~m}$, (ii) the height of the domain was increased vertically from 325 to $1725 \mathrm{~m}$, and (iii) given no effect on potentials, the domain was enlarged by a factor of more than two, where $R=2000 \mathrm{~m}$ and $H=4125 \mathrm{~m}$. The model assumed a borehole of $8^{\prime \prime}$ diameter $(20.3 \mathrm{~cm})$ and $2125 \mathrm{~m}$ length centered around the electrode $\mathrm{A}_{0}$ 
(Fig. 6b). The numerical model of this geometry gave 746020 finite elements. Within elements, electric potentials were approximated by a quadratic function, giving 1.5 million total degrees of freedom to be solved. For a given depth, solutions required approximately 1 min CPU time and less than 4 GB memory on an Intel Server.

In practice, the apparent resistivity is calculated from the potential difference between the electrode $M_{1}$ and the reference electrode

$$
R_{a}^{\mathrm{LLD}}=K^{\mathrm{LLD}} \frac{V_{\mathrm{M} 1}-V_{\mathrm{N}}}{I_{0}},
$$

where the geometric factor of the device with a finite extension, $K^{\mathrm{LLD}}$, is unknown. The geometry of the device (long and adjacent electrodes) precludes accurate pointwise approximation. The electric potential for electrodes $\mathrm{M}_{1}$ and $\mathrm{N}$ in a homogeneous geologic medium with true resistivity of $R_{t}=R_{m}=$ $0.1,1,10$, and $100 \mathrm{ohm} \mathrm{m}$ at $I_{0}=1 \mathrm{~A}$ was calculated to determine the geometric factor,

$$
K^{\mathrm{LLD}}=R_{t} \frac{I_{0}}{V_{\mathrm{M} 1}-V_{\mathrm{N}}} \cong 0.90693 \mathrm{~m} .
$$

This calculation yielded a geometric coefficient, $K^{\mathrm{LLD}}=0.90693 \mathrm{~m}$, that recovered the true resistivity values to within a standard deviation of less than $0.3 \%$.

Numerical modeling was also used to investigate the effects of borehole mud on apparent resistivity. The diameter of the borehole and the mud resistivity was fixed $\left(d=0.20 \mathrm{~m}\right.$ and $\left.R_{m}=1 \mathrm{ohm} \mathrm{m}\right)$, while the true resistivity varied from $R_{t}=0.1$ to $R_{t}=1000 \mathrm{ohm} \mathrm{m}$. Figure 7 shows potential field and current line structure for three different cases. At low $R_{t}$, elevated potential was localized near current electrodes. As $R_{t}$ increased, areas of elevated
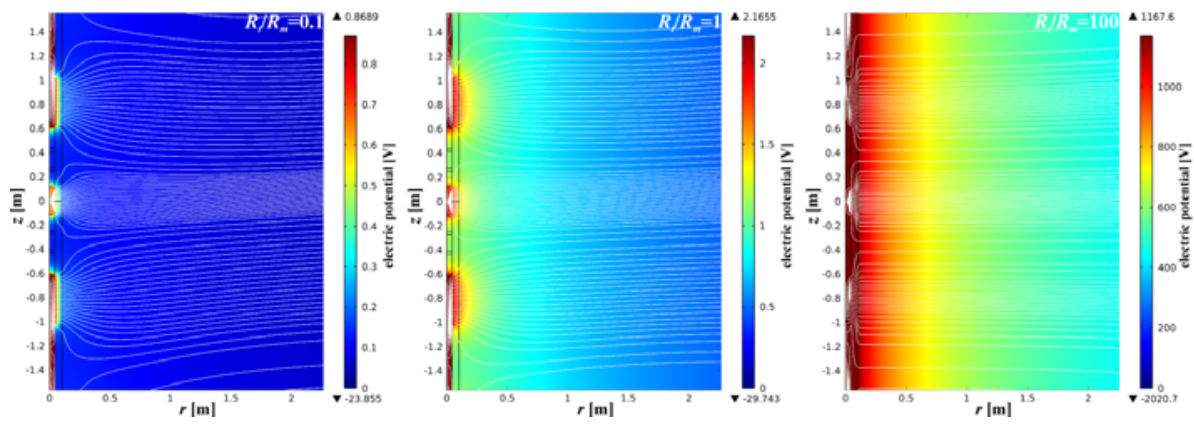

Fig. 7. The electric potential field (colored) and current line distribution (white lines) around middle and guard electrodes at different ratios for true resistivity and mud resistivity $\left(R_{t} / R_{m}=0.1 ; 1 ; 100\right)$. 


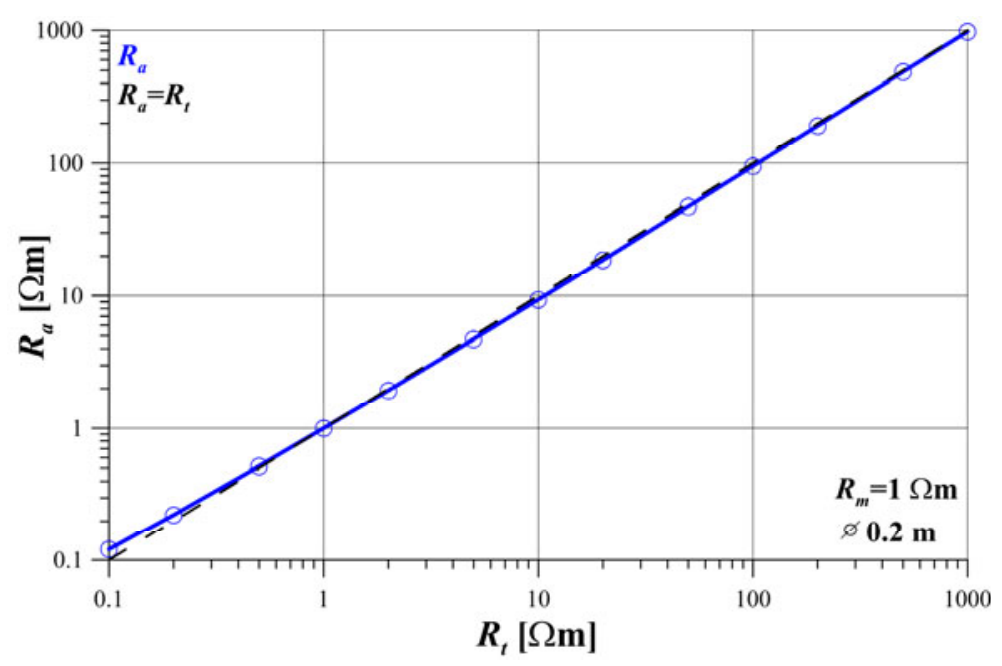

Fig. 8. Relation between the apparent and true resistivity in the presence of a borehole mud having $R_{m}=1 \mathrm{ohm} \mathrm{m}$; borehole diameter $d=0.20 \mathrm{~m}$.

potential became more elliptical, encircling the whole device. Current lines become more perpendicular to the borehole due to focusing which ensures the deep penetration of the LLD device. Figure 8 demonstrates that the device focused in LLD mode was basically insensitive to mud in the borehole. The apparent resistivity approximates true resistivity, with small deviation observed only at very low $R_{t}$ values.

Further testing demonstrated device performance at horizontal layer boundaries. Figure 9a displays the inhomogeneous current density and current line distribution when the true resistivity of the upper layer is larger by 2 orders of magnitude and the boundary is at the middle of $\mathrm{A}_{0}$. The modeled $\log$ (Fig. 9b) confirms that the apparent resistivity approximates true resistivity with some distance from the boundary (in the presence of mud, $R_{a}$ is lower than $R_{t}$ ). Global overshoot occurs just above the boundary in the absence of a borehole while a local overshoot occurs in the case of a mud filled borehole. The Groningen effect is observable as an apparent resistivity increase beneath the boundary when the reference potential electrode, $\mathrm{N}$, is positioned at the resistive layer (the distance between $\mathrm{A}_{0}$ and $\mathrm{N}$ is $25 \mathrm{~m}$ ). It is worth noting that the two curves (blue and red) converge in the lower layer where $R_{m}=R_{t}=1 \mathrm{ohm} \mathrm{m}$.

A final set of numerical tests investigated the effects of a thin, high resistivity layer on $R_{a}$ (Fig. 10). The layer was assigned a thickness of $12 \mathrm{~m}$ (about half of the distance separating $\mathrm{A}_{0}$ and $\mathrm{N}$ electrodes) and a resistivity exceeding the shoulder bed resistivity by two orders of magnitude. Apparent 
a

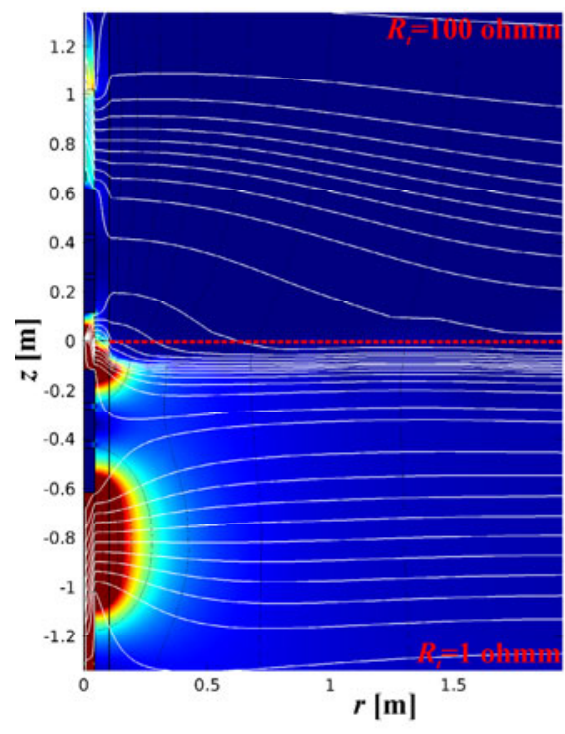

b

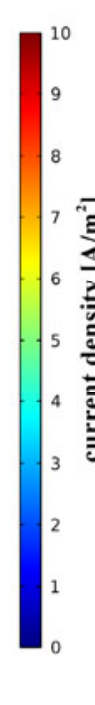

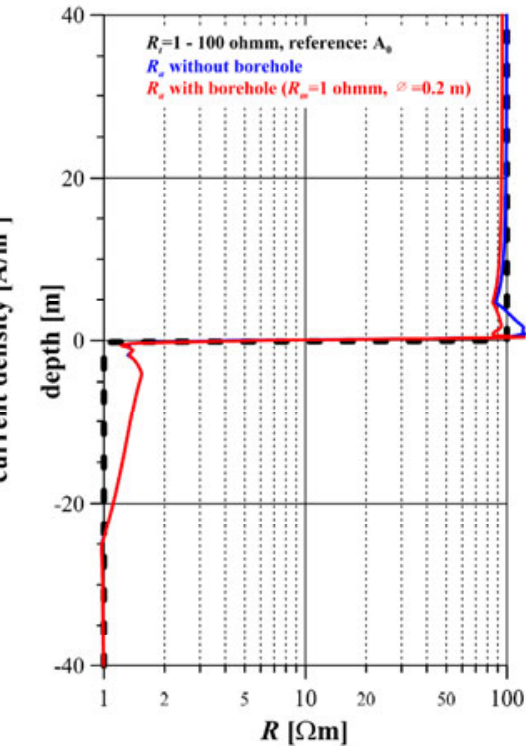

Fig. 9: (a) Current density and current line distribution in the vicinity of the electrodes when $\mathrm{A}_{0}$ is opposite to the layer boundary separating the layers with high (up) and low (down) resistivity; (b) Relation of the apparent and true resistivity logs across the layer boundary. Red line denotes the apparent resistivity log with borehole, blue line without borehole, and black dashed line gives the true resistivity.

resistivity maxima appear above and below the bed. The stratigraphically lower maximum is of greater magnitude due to the Groningen effect. Within the bedded layer, $R_{a}$ is lower than $R_{t}$ due to effect of the thin layer. The apparent maxima in the presence of low resistivity mud are localized at the boundaries.

The apparent resistivity curve calculated using the Coulomb's charges method, assuming a $0.2 \mathrm{~m}$ diameter borehole and $1 \mathrm{ohm} \mathrm{m}$ mud resistivity, was compared with results calculated by the ELTE method (Fig. 10). The curves are visibly similar but theoretical resistivity and obtained apparent resistivity also differ in several important ways. Generally, ELTE results obtained with borehole are closer to the theoretical resistivity model. Differences can be attributed to the different physical approaches used by each method, errors related to approximations and calculation methods and model uncertainties. Differences between theoretical and calculated values become distinctly visible at points where there are large shifts in resistivity (Fig. 10). Calibration curves calculated by the Coulomb's charges method cause diverging results for apparent and true resistivity (Cichy and Ossowski 

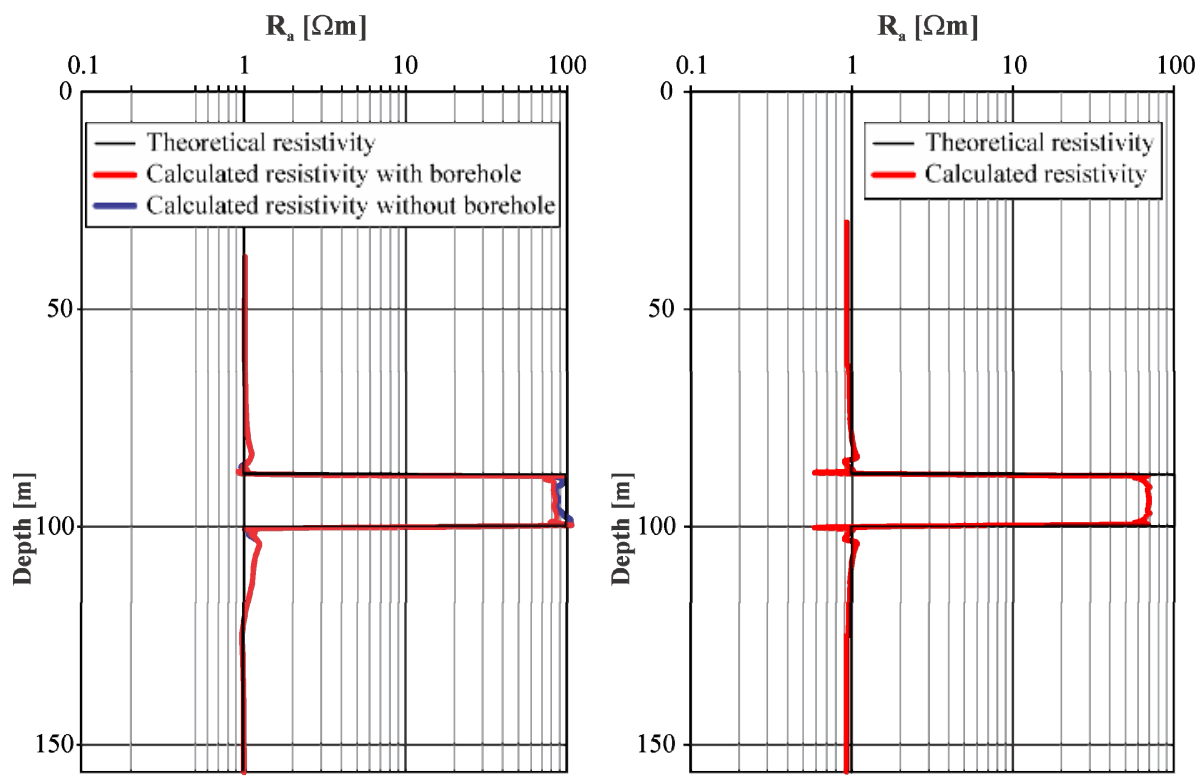

Fig. 10. Comparison of apparent and true resistivity logs across a thin, resistive layer. The left panel shows ELTE model results for apparent resistivity with the borehole in red and without the borehole in blue, and the true resistivity of the strata shown by the black line. The right panel shows Coulomb's charges method results for the apparent resistivity log with borehole in red and the true resistivity of the strata in black.

2015) (Fig. 3). The lower $K^{\mathrm{LLD}}$ value of $0.81 \mathrm{~m}$ (relative to $K^{\mathrm{LLD}}=$ $0.90693 \mathrm{~m}$ from ELTE method) gave lower apparent resistivity for Coulomb's charges method results. For a bed having a true resistivity of $100 \mathrm{ohm} \mathrm{m}$, the Coulomb's charges method gave $78.53 \mathrm{ohm} \mathrm{m}$. The apparent resistivity curve (Fig. 10 right) is perfectly symmetric and did not exhibit Groningen effects because of how the methods summed potential generated by normal device components.

\section{COMPARISON OF METHODS USING GEOLOGIC DATA}

Table 2 describes a stratigraphic section typical of the Fore-Sudetic Monocline in southwestern Poland, constructed from geological data and well logs.

The profile was used to compare results obtained from the Coulomb's charges and ELTE methods. A key unit in the profile was the Main Dolomite (cyclothem Z2, Stassfurt), which is a porous, hydrocarbon- or watersaturated carbonate overlain (in succession) by the Basal Anhydrite, Older Salt, and Main Anhydrite (Table 2). The thickness of the Main Dolomite car- 
Table 2

Geological profile typical of the Fore-Sudetic Monocline, southwestern Poland

\begin{tabular}{|c|c|c|c|c|c|c|}
\hline $\begin{array}{c}\text { Strata } \\
\text { I.D. }\end{array}$ & $\begin{array}{l}\text { Top } \\
{[\mathrm{m}]}\end{array}$ & $\begin{array}{l}\text { Bot- } \\
\text { tom } \\
{[\mathrm{m}]}\end{array}$ & \begin{tabular}{|c|} 
Thick- \\
ness \\
{$[\mathrm{m}]$} \\
\end{tabular} & Lithology & $\begin{array}{c}\text { Age and/or } \\
\text { stratigraphic name }\end{array}$ & \begin{tabular}{|l} 
Resisti- \\
vity \\
[ohm m]
\end{tabular} \\
\hline 1 & 0 & 978 & 978 & & $\begin{array}{l}\text { Quaternary, Tertiary, } \\
\text { Jurassic }\end{array}$ & \\
\hline 2 & 978 & 1966 & 988 & $\begin{array}{l}\text { claystone, sandstone, } \\
\text { mudstone }\end{array}$ & Upper Triassic & \\
\hline 3 & 1966 & 2237 & 271 & \begin{tabular}{|l|} 
limestone, \\
dolomitic limestone, \\
marly claystone, \\
dolomitic claystone \\
\end{tabular} & $\begin{array}{l}\text { Middle Triassic, } \\
\text { Muschelkalk }\end{array}$ & 150 \\
\hline 4 & 2237 & 2349 & 112 & \begin{tabular}{|l|} 
claystone, limestone, \\
dolomitic limestone, \\
marl
\end{tabular} & $\begin{array}{l}\text { Upper Bunter } \\
\text { Sandstone, Red }\end{array}$ & 100 \\
\hline 5 & 2349 & 2576 & 227 & $\begin{array}{l}\text { claystone, mudstone, } \\
\text { sandstone }\end{array}$ & $\begin{array}{l}\text { Middle Bunter } \\
\text { Sandstone }\end{array}$ & $60-80^{\#}$ \\
\hline 6 & 2576 & 2905 & 329 & \begin{tabular}{|l|} 
claystone, \\
lime-claystone, \\
dolomitic claystone, \\
sandstone, mudstone \\
\end{tabular} & $\begin{array}{l}\text { Lower Bunter } \\
\text { Sandstone }\end{array}$ & 100 \\
\hline 7 & 2905 & 2932 & 27 & claystone & Transient Claystones & 30 \\
\hline 8 & 2932 & 2980 & 48 & halite & The Youngest Salt & 2000 \\
\hline 9 & 2980 & 2994 & 14 & anhydrite and clay & $\begin{array}{l}\text { Pegmatite Anhydrite } \\
(1.5 \mathrm{~m}), \text { Red Clay }\end{array}$ & 20 \\
\hline 10 & 2994 & 3051 & 57 & halite & Younger Salt & 2000 \\
\hline 11 & 3051 & 3059 & $8^{*}$ & $\begin{array}{l}\text { anhydrite, gray clay, } \\
\text { anhydrite }\end{array}$ & \begin{tabular}{|l|} 
Main Anhydrite, \\
Gray, Salt Clay, \\
Covering Anhydrite
\end{tabular} & 2000 \\
\hline 12 & 3059 & 3297 & 238 & halite & Older Salt & 2000 \\
\hline 13 & 3297 & 3303 & 6 & anhydrite & Basal Anhydrite & 2000 \\
\hline 14 & 3303 & 3307 & $4 *$ & $\begin{array}{l}\text { dolomite } \\
\text { (up to } 60 \mathrm{~m} \text { thick) }\end{array}$ & $\begin{array}{l}\text { Main Dolomite, Z2, } \\
\text { Stassfurt }\end{array}$ & $40-150^{\#}$ \\
\hline 15 & 3307 & 3349 & 42 & anhydrite & Upper Anhydrite & 2000 \\
\hline 16 & 3349 & 3370 & 21 & halite & The Oldest Salt & 2000 \\
\hline 17 & 3370 & 3518 & 148 & anhydrite & Lower Anhydrite & 2000 \\
\hline 18 & 3518 & $\begin{array}{c}3519 . \\
9\end{array}$ & $1^{*}$ & $\begin{array}{l}\text { limestone } \\
\text { (up to } 80 \mathrm{~m} \text { thick) }\end{array}$ & one & $0^{\#}$ \\
\hline 19 & 3519 & 3520 & 1 & shale & Copper Shale & 5 \\
\hline 20 & 3520 & 3652 & 132 & sandstone & Rotliegend & $10-30$ \\
\hline
\end{tabular}

*) thickness of unit may differ in different wells, \#) range of values observed for the unit. 


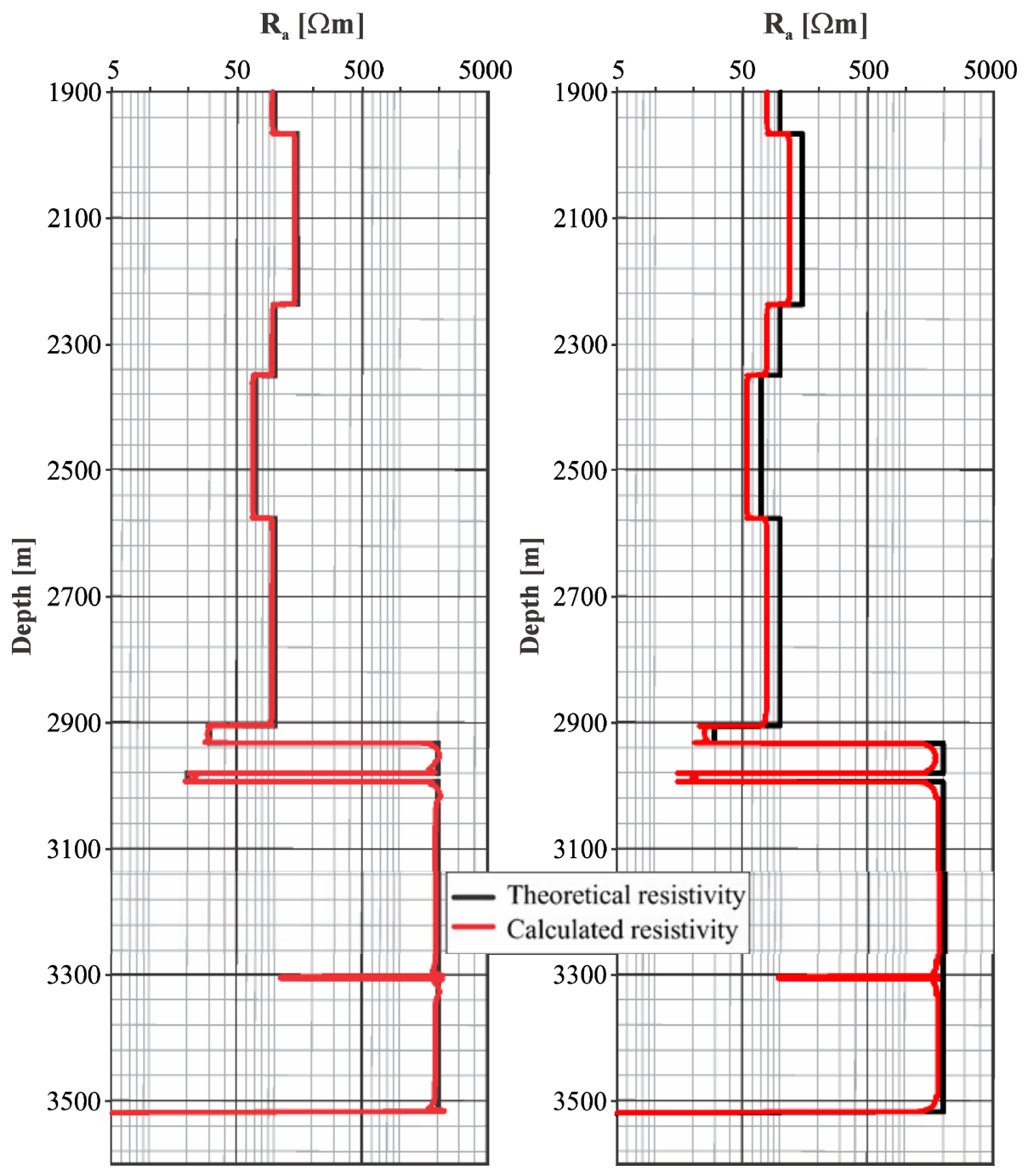

Fig. 11. Calculations and modeling results for apparent resistivity of a constructed geologic profile. Theoretical resistivity model is shown in black and calculated resistivity is shown in red. The left panel shows ELTE model results and the right panel shows Coulomb's charges results.

bonate varied, as did the thick, overlying accumulation of the Basal Anhydrite, Older Salt, and Main Anhydrite. This succession of strata was key to our analysis due to the challenges it posed for accurate resistivity recording.

The calculated and modeled results are visibly similar (Fig. 11). A lower assumed $K^{\mathrm{LLD}}$ caused lower apparent resistivity results for the Coulomb's 
charges method. A bed having a true resistivity of $2000 \mathrm{ohm} \mathrm{m}$ gave a calculated apparent resistivity of $1811 \mathrm{ohm} \mathrm{m}$.

\section{FURTHER APPLICATION OF MODELING RESULTS}

Comparison of the apparent resistivity curves demonstrated that both methods provide similar results and may be used as a numerical tool for determining and interpreting apparent dual laterolog resistivity. Calculated and theoretical resistivity values were obtained by the Coulomb's charges method using the assumed resistivity values $R_{t}, R_{x o}, R_{m}$ and geometrical parameters $(D / d)$ shown in Table 3.

Table 3

Input values for theoretical resistivity calculations

\begin{tabular}{|l|l|l|r|r|r|r|r|}
\hline$D / d$ & $R_{x o} / R_{m}$ & $R_{t} / R_{m}$ & $D / d$ & $R_{x o} / R_{m}$ & $R_{t} / R_{m}$ & $R_{x o} / R_{m}$ & $R_{t} / R_{m}$ \\
\hline 1 & 2 & 3 & 4 & 5 & 6 & 7 & 8 \\
1.5 & 1 & 1 & 12 & 10 & 10 & 250 & 250 \\
2 & 1.25 & 1.25 & 14 & 15 & 15 & 300 & 300 \\
2.5 & 1.5 & 1.5 & 16 & 20 & 20 & 400 & 400 \\
3 & 1.75 & 1.75 & 24 & 30 & 30 & 500 & 500 \\
4 & 2 & 2 & 32 & 40 & 40 & 1000 & 1000 \\
5 & 2.5 & 2.5 & & 50 & 50 & 2000 & 2000 \\
6 & 3 & 3 & & 75 & 75 & 3000 & 3000 \\
7 & 4 & 4 & & 100 & 100 & 5000 & 5000 \\
8 & 5 & 5 & & 150 & 150 & 7500 & 7500 \\
10 & 7.5 & 7.5 & & 200 & 200 & 10000 & 10000 \\
\hline
\end{tabular}

Explanations: $R_{t}$ - true resistivity of unit, $R_{m}$-mud resistivity, $R_{x o}$ - invaded zone resistivity, $d$ and $D$ - respective radii for borehole and invaded zone; columns 7 and 8 represent continuations of columns 5 and 6 , respectively.

The results, along with analysis of discrepancies and uncertainty, suggest a processing methodology for dual laterolog apparent resistivity (Fig. 12). The method determines the true resistivity of the virgin and invaded zones together with invaded zone diameter, $D$. The proposed methodology implements corrections in a manner similar to methods used by Halliburton and Schlumberger (www.halliburton.com; www.slb.com). It consists of two linear corrections in sequence, (i) for borehole influence, and (ii) influence of the interpreted bed thickness and shoulder bed resistivity. A third correction, (iii) removes filtration effects according to tornado charts. Corrections may be implemented in an automatic or semiautomatic fashion (in the case of thickness and shoulder bed resistivity). 


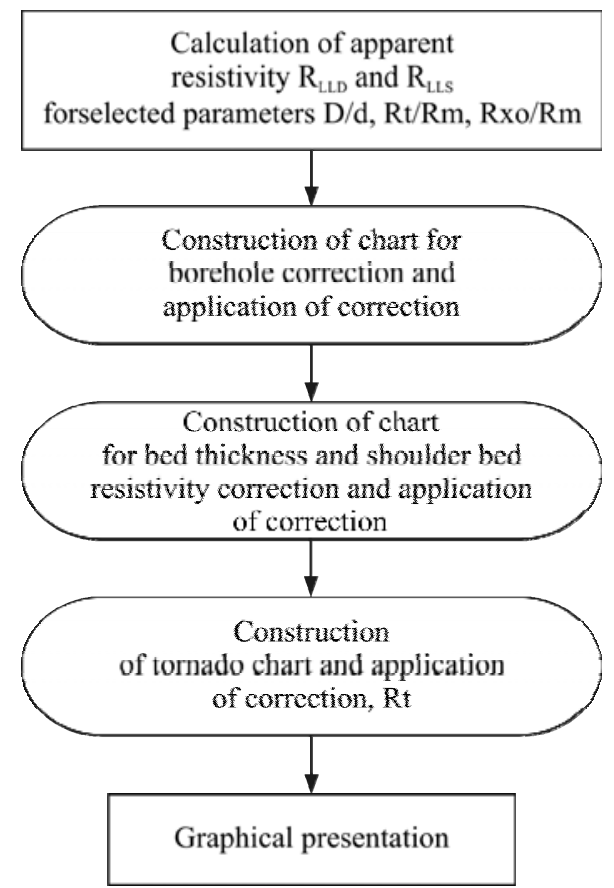

Fig. 12. Flow chart of apparent resistivity correction procedure. Dual laterolog apparent resistivity is recorded and interpreted to determine the true resistivity of the virgin zone.

A calculation based on the Coulomb's charges method was used to compute apparent resistivity for various resistivity conditions and geometric models (Table 3). The data were also used to construct nomograms for comparison with established reference charts for apparent resistivity borehole corrections used by Halliburton (Fig. 13). Values in Fig. 13 show that the correction procedures described here give the same results for borehole corrected resistivity as those given by Halliburton reference charts.

Charts for bed thickness and shoulder bed resistivity corrections were also constructed using the dataset in Table 3 (Fig. 14). Similar modeling of efficient corrections for layer thickness and shoulder bed resistivity was performed for well logging in horizontal wells (Shaogui et al. 2009).

A third type of correction for filtration effects in the reservoir rock (i.e., tornado charts) was also applied using the dataset in Table 3. Equation 19 was used to select suitable data and construct tornado charts:

$$
R_{t} / R_{\mathrm{LLD}}=f\left[\log \left(\frac{R_{\mathrm{LLD}}}{R_{\mathrm{LLS}}} ; \frac{R_{\mathrm{LLD}}}{R_{R_{x o}}}\right)\right] .
$$




\section{LLD}
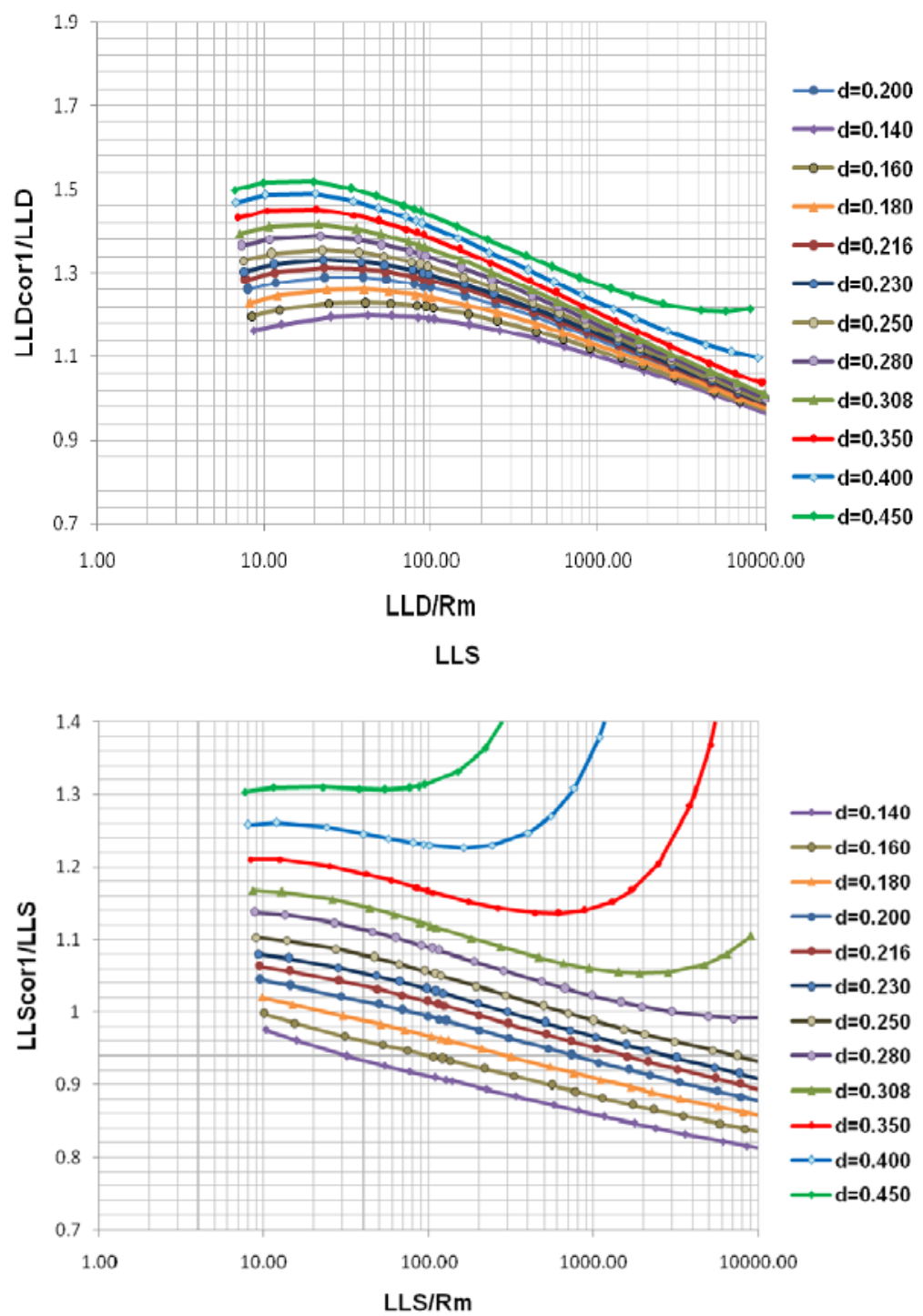

Fig. 13. Borehole correction charts for LLD (upper panel) and LLS (lower panel) resistivity.

Figure 15 gives an example of a tornado chart based on an invaded zone and mud resistivity ratio of $R_{x o} / R_{m}=100\left(R_{m}=1 \mathrm{ohm} \mathrm{m}\right)$. This apparent resistivity correction procedure uses two sets of curves with parameters $R_{t} / R_{m}$ and $D / d$ to determine true resistivity, $R_{t}$. The initial point of the tornado 

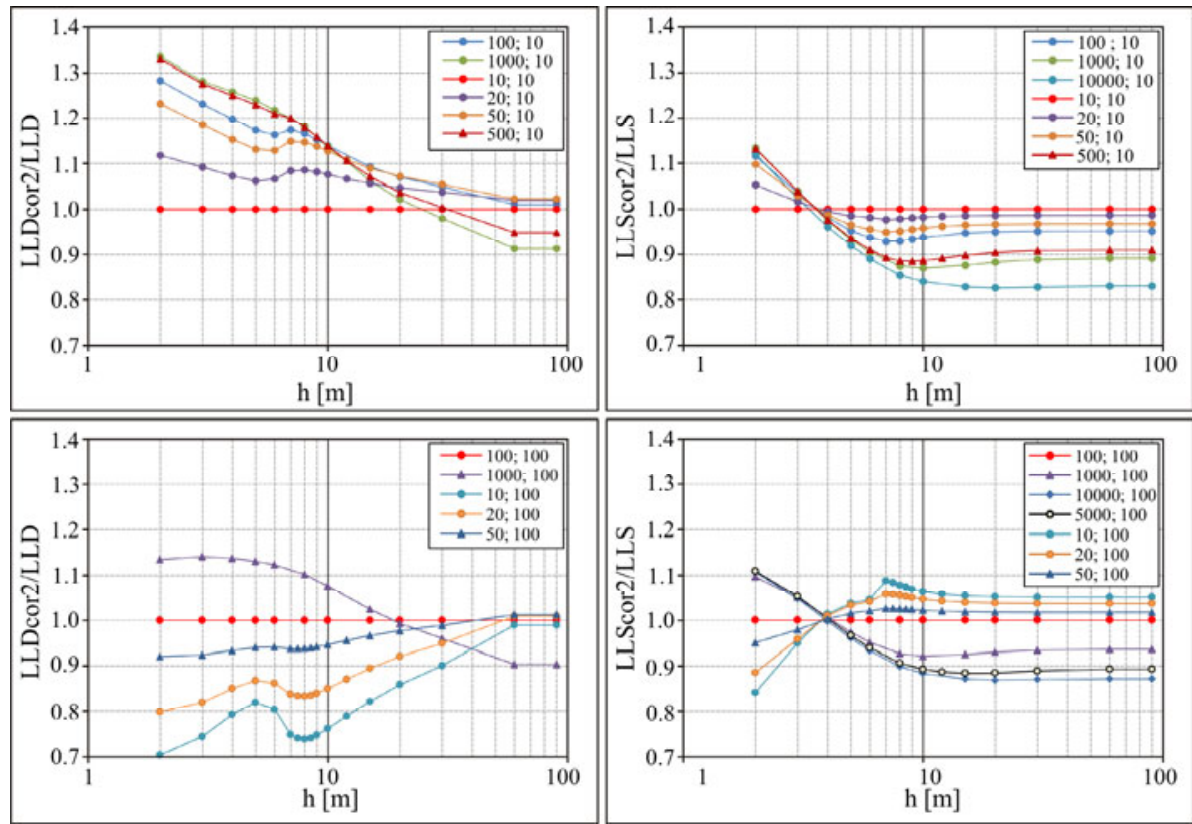

Fig. 14. Example of thickness and shoulder bed resistivity correction charts for LLD (left panel) and LLS (right panel) resistivity; the first number in legends means $R_{t}$, the second $R_{s}$.

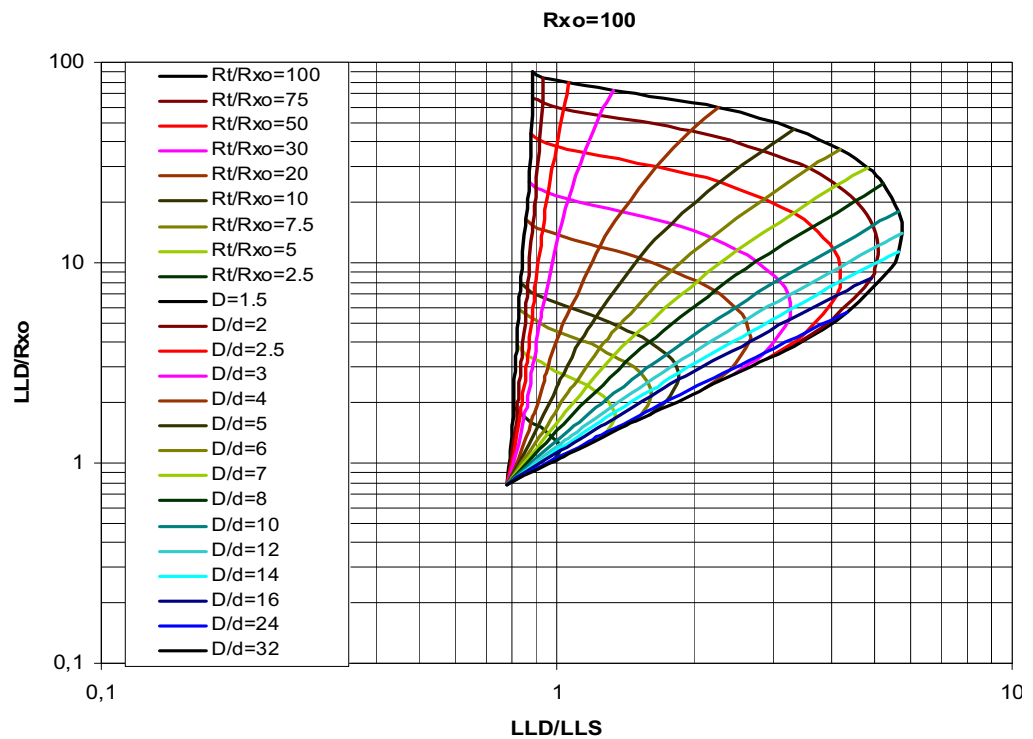

Fig. 15. Example of a tornado chart: $R_{t}$ - true resistivity, $R_{x o}-$ invaded zone resistivity, LLD and LLS refer to deep and shallow laterolog operating modes, $d-$ borehole diameter, $D$ - invaded zone diameter. 


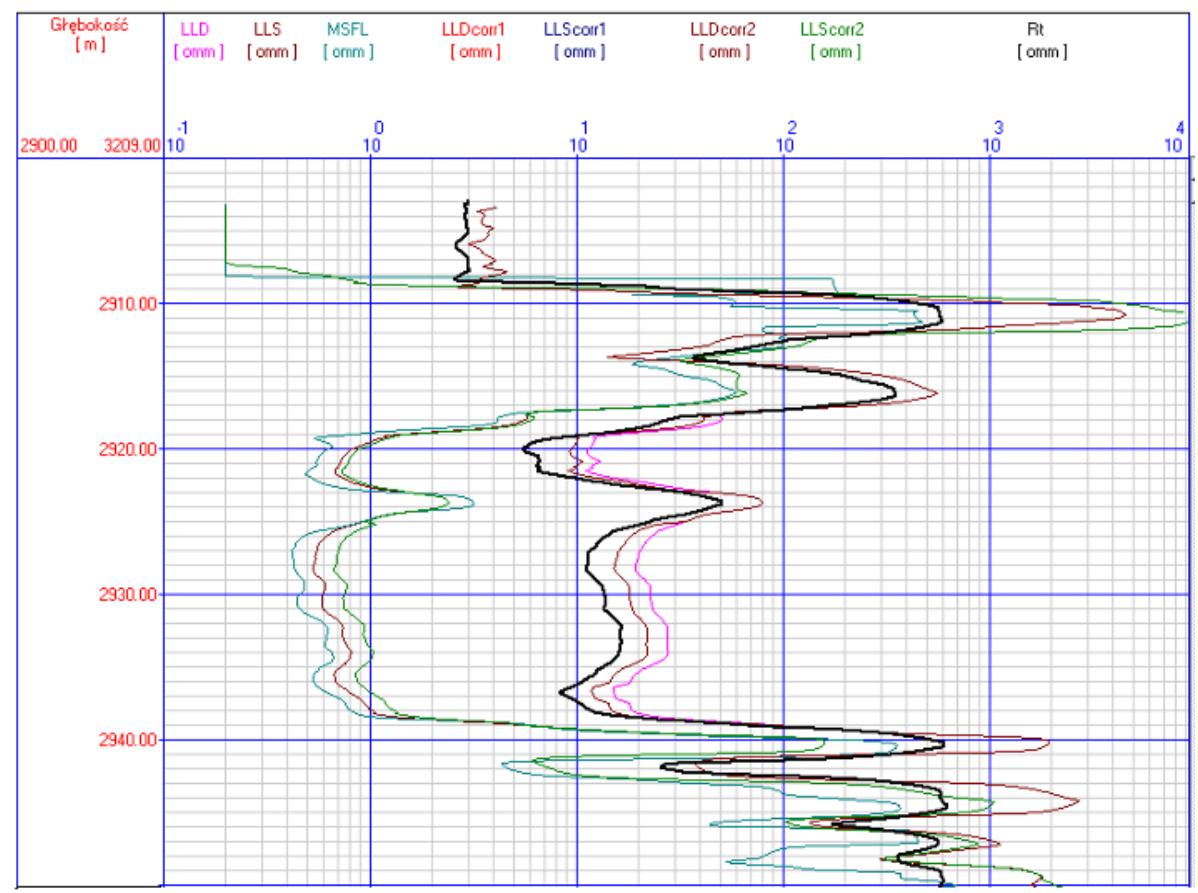

Fig. 16. Resistivity correction procedure applied to actual geological data according flow chart (Fig. 12; W5 borehole, Main Dolomite section of the Fore-Sudetic Monocline). Primary apparent resistivity is shown by LLD (pink), LLS (brown), and MSFL (forest green). Resistivity after borehole correction is shown by LLDcorr1 (red). Resistivity after layer thickness and shoulder bed correction is shown by LLDcorr2 (brown) and LLScorr2 (green). All corrections were applied to the true resistivity $R_{t}$ (black) including tornado chart adjustments.

curves shifts visibly due to a discrepancy in the calculated apparent resistivity and theoretical resistivity of the model (Cichy and Ossowski 2015). Coordinates of this point are known from calculated results and are used in the final step of the correction procedure.

The apparent resitivity correction procedure gave results that, like true resistivity, were less than apparent resistivity values. Figure 16 demonstrates the influence of applied corrections. This example was based on well log data from well W5 located in the Fore-Sudetic Monocline of southwestern Poland. This part of the stratigraphic section includes the Main Dolomite (2912.0-2964.0 m), which shows significant variation in resistivity. The upper part of the Main Dolomite (beneath the Basal Anhydrite, up to $2918.5 \mathrm{~m}$ ) is highly resistive, the middle part exhibits a relatively low resistivity, and the bottom part is highly resistive. The casing shoe is located at $2908.0 \mathrm{~m}$ depth. The upper part of borehole was cased with steel pipes of 7" diameter. 
Processing steps addressed apparent resistivity of LLD and LLS operating modes of the devices. The first step applied a semiautomatic correction for influence of bed thickness and shoulder bed resistivity (borehole correction performed by a service company). The invasion effect was then reduced by the tornado chart. All corrections were performed using software dedicated to the proposed solution and according to flow chart sequence (Fig. 12). The corrections generated lower resistivity values (Fig. 16).

\section{CONCLUSIONS}

This paper adds two methods to the body of procedures used to correct laterolog resistivity. Both methods provide comparable results using modern numerical tools to calculate apparent resistivity in complex geologic media and geometric situations. The methodology derives true resistivity from apparent resistivity corrections of DLT-GA laterolog data and provides a flexible approach that can also be adapted to specific cases.

Acknowledgments. Results presented in the paper were partially obtained from a 2012-2014 project financed by POGC Warsaw, Poland, at AGH University of Science and Technology, Kraków, Poland. Authors thank POGC, Warsaw, Poland, for providing technical information and relevant data necessary for testing the solutions. The paper was partially financed by statutory funds of the AGH UST Faculty of Geology Geophysics and Environment Protection, Department of Geophysics, Kraków, Poland, project No. 11.11.140.769, 2014.

\section{References}

Alpin, L.M. (1964), Some theoretical method for solving direct resistivity logging problem, Izv. AN SSSR, Geof., 2.

Alpin, L.M., D.S. Dajev, and A.D. Karinskij (1985), The Theory of Alternating Fields for Exploratory Geophysics, Niedra, Moskva.

Anderson, B.I. (2001), Modeling and inversion methods for the interpretation of resistivity logging tool response, Ph.D. Thesis, Delft University of Technology, Delft, The Netherlands.

Chew, W.C., Z. Nie, Q.-H. Liu, and B. Anderson (1991), An efficient solution for the response of electrical well logging tools in a complex environment, IEEE Trans. Geosci. Remote Sens. 29, 2, 308-313, DOI: 10.1109/36.73673. 
Cichy, A., and A. Ossowski (2015), Modeling electrical field distribution in layered geological rock formations with a borehole using the Coulomb charges method, Acta Geophys. 63, 5, 1244-1255, DOI: 10.2478/s11600-014-0253-2.

Davydycheva, S., V. Druskin, and T. Habashy (1996), Solution of Maxwell's equation in an arbitrary 3-D inhomogeneous anisotropic media with sharp discontinuities using a finite-difference scheme on a regular Cartesian grid with material averaging, Tech. Report EMG-002-96-15, SchlumbergerDoll Research Center, Cambridge, USA.

Drahos, D. (1984), Electrical modeling of the inhomogeneous invaded zone, Geophysics 49, 10, 1580-1585, DOI: 10.1190/1.1441566.

Drahos, D., and A. Galsa (2007), Finite element modeling of penetration electric sonde, Magy. Geofiz. 48, 1, 22-30 (in Hungarian).

Ellis, D.V., and J.M. Singer (2007), Well Logging for Earth Scientists, 2nd ed., Springer, Dordrecht.

Halliburton (1992), Technical manual of dual laterolog DLT-GA device, Halliburton Co., Houston, USA, http://www.halliburton.com.

Lovell, J.R. (1993), Finite element methods in resistivity logging, Ph.D. Thesis, Delft University of Technology, Delft, The Netherlands.

Moran, J., and J. Timmons (1957), The mathematical theory of ring electrode laterolog response in thick-beds, Project RR-602, Schlumberger-Doll Research Center, Cambridge, USA.

Moskow, S.,V. Druskin, T. Habashy, P. Lee, and S. Davydycheva (1999), A finite difference scheme for elliptic equations with rough coefficients using a Cartesian grid nonconforming to interfaces, SIAM J. Numer. Anal. 36, 2, 442-464, DOI: 10.1137/S0036142997318541.

Nam, M.J., D. Pardo, and C. Torres-Verdín (2010), Assessment of Delaware and Groningen effects on dual-laterolog measurements with a self-adaptive hp finite-element method, Geophysics 75, 6, F143-F149, DOI: 10.1190/ 1.3496670 .

Pardo, D., C. Torres-Verdin, and L. Demkowicz (2005), Self-adaptive goal-oriented $\mathrm{hp}$-finite element simulations of induction and laterolog measurements in the presence of steel casing. In: Proc. Fifth Annual Meeting "Joint Industry Research Consortium on Formation Evaluation”, 1 August 2005, The University of Texas, Austin, USA.

Ribeiro, M., and A. Carrasquilla (2013), New approach to calculate the mud invasion in reservoirs using well logs. In: Proc. 13th Int. Congr. Brazilian Geophysical Society and EXPOGEF, 26-29 August 2013, Rio de Janeiro, Brazil.

Shaogui, D., L. Zhuqiang, and L. Zhiqiang (2009), Response of dual laterolog and fast correction for layer thickness and shoulder bed in horizontal wells, Petrol. Explor. Develop. 36, 6, 725-729, DOI: 10.1016/S1876-3804(10)60005-5. 
Trouiller, J.C., and I. Dubourg (1994), A better deep laterolog compensated for Groningen and reference effects. In: SPWLA 35th Annual Logging Symposium, 19-22 June 1994, Tulsa, USA, SPWLA-1994-VV.

Wang, H.M., L.C. Shen, and G.J., Zhang (1998), Dual laterolog response in 3-D environments. In: SPWLA 39th Annual Logging Symposium, 26-28 May 1998, Keystone, USA, SPWLA-1998-X.

Received 28 January 2015 Received in revised form 7 April 2015

Accepted 10 April 2015 